\title{
The Application of Principal Component Analysis Using Fixed Eigenvectors to the Infrared Thermographic Inspection of the Space Shuttle Thermal Protection System
}

\author{
by K. Elliott Cramer and William P. Winfree \\ National Aeronautics and Space Administration, Langley Research Center, Hampton, \\ VA, USA
}

\begin{abstract}
The Nondestructive Evaluation Sciences Branch at NASA's Langley Research Center has been actively involved in the development of thermographic inspection techniques for more than 15 years. Since the Space Shuttle Columbia accident, NASA has focused on the improvement of advanced NDE techniques for the Reinforced Carbon-Carbon (RCC) panels that comprise the orbiter's wing leading edge. Various nondestructive inspection techniques have been used in the examination of the RCC, but thermography has emerged as an effective inspection alternative to more traditional methods. Thermography is a non-contact inspection method as compared to ultrasonic techniques which typically require the use of a coupling medium between the transducer and material. Like radiographic techniques, thermography can be used to inspect large areas, but has the advantage of minimal safety concerns and the ability for single-sided measurements.

Principal Component Analysis (PCA) has been shown effective for reducing thermographic NDE data. A typical implementation of PCA is when the eigenvectors are generated from the data set being analyzed. Although it is a powerful tool for enhancing the visibility of defects in thermal data, PCA can be computationally intense and time consuming when applied to the large data sets typical in thermography. Additionally, PCA can experience problems when very large defects are present (defects that dominate the field-of-view), since the calculation of the eigenvectors is now governed by the presence of the defect, not the "good" material. To increase the processing speed and to minimize the negative effects of large defects, an alternative method of PCA is being pursued when a fixed set of eigenvectors is used to process the thermal data from the RCC materials. These eigen vectors can be generated either from an analytic model of the thermal response of the material under examination, or from a large cross section of experimental data.

This paper will provide the details of the analytic model; an overview of the PCA process; as well as a quantitative signal-to-noise comparison of the results of performing both embodiments of PCA on thermographic data from various RCC specimens.

Details of a system that has been developed to allow insitu inspection of a majority of shuttle RCC components will be presented along with the acceptance test results for this system. Additionally, the results of applying this technology to the Space Shuttle Discovery after its return from flight will be presented.
\end{abstract}




\section{Introduction}

The space shuttle's thermal protection system is made from materials that can perform in temperatures between $-150^{\circ} \mathrm{C}$ to $1650^{\circ} \mathrm{C}$. Reinforced carbon-carbon (RCC) is used in areas where temperatures exceed $1275^{\circ} \mathrm{C}$. This gray colored material is located at the wing leading edges, T-seals, nose cap, and chin panel. It is made from layers of rayon cloth graphitized and impregnated with a phenolic resin and coated with silicon-carbide. Locations on the shuttle that experience temperatures below $1275^{\circ} \mathrm{C}$ are protected with black tiles made of low-density, highpurity silica amorphous fiber insulation. These high-temperature reusable surface insulation tiles (HRSI) are found on the underside of the vehicle where the RCC material is not used. Additionally, low-temperature reusable surface insulation white tiles (LRSI) are used in areas of the fuselage where temperatures do not exceed $650^{\circ} \mathrm{C}$. These tiles differ from their black counterparts in that they are thinner [[1]]. Fig. 1 shows the location of the RCC materials on the orbiter.

According to the Columbia Accident Investigation Board the loss of the Space Shuttle Columbia and its crew on February 1, 2003 was due to a breach in the thermal protection system on the leading edge of the orbiter's left wing. It was concluded that the breach was a result of an impact with insulating foam that had fallen off from the external fuel tank 81 seconds into the launch. Although RCC material has shown excellent mechanical qualities in harsh environments over the past twenty years, enhanced inspections since 1997 have revealed some damage. The Board recommended that a comprehensive inspection plan be implemented to determine the structural integrity of the RCC using the most advanced nondestructive inspection techniques available [[2]].

Full-scale foam ballistic impact tests on wing leading edge RCC panels have been conducted over the past months. Various nondestructive inspection techniques have been used in the examination of the RCC material post impact. Thermography has proven to be an effective inspection tool over more traditional methods. It is a non-contact inspection method as compared to ultrasonic techniques which typically require the use of a coupling medium between the transducer and material. Like radiographic techniques, thermography can be used to inspect large areas, but has the advantage of minimal safety concerns and the ability for single-sided measurements. 


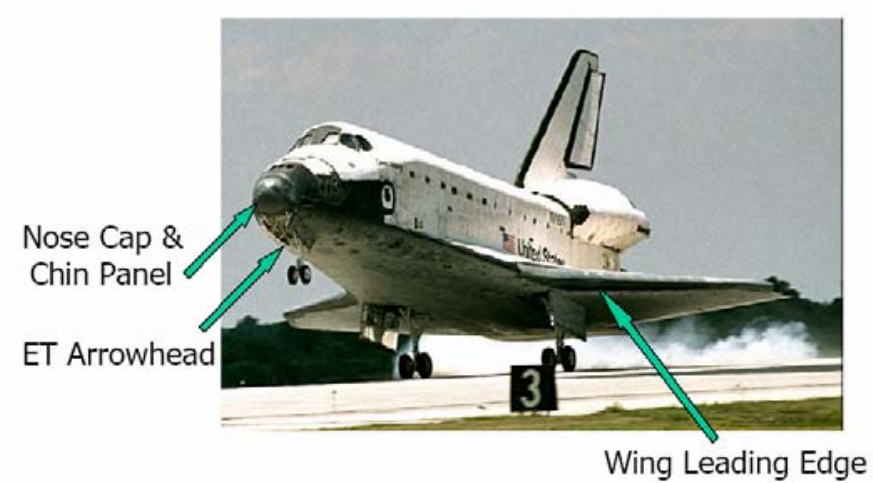

Fig. 1. Locations of the RCC components of the thermal protection system on the space shuttle.

Principal Component Analysis has been effectively used as an analysis tool for a number of thermography applications [[3]-[5]]. Although it is a powerful tool for enhancing the visibility of defects in thermal data, PCA can be computationally intense and time consuming on the large data sets typical in thermography. Additionally, PCA can experience problems when very large defects are present (defects that dominate the field-of-view), since the calculation of the eigen vectors is now governed by the presence of the defect, not the "good" material. To increase the processing speed and to minimize the negative effects of large defects, an alternative method of PCA is being pursued when a fixed set of eigen vectors, generated from an analytic model of the thermal response of the material under examination, is used to process the thermal data from the RCC materials.

\section{Experimental setup}

For application to RCC materials, NASA is currently using the commercial infrared thermography system EchoTherm ${ }^{\circledR}$ manufactured by Thermal Wave Imaging, Inc. The IR imager is a commercial radiometer with a cooled $256 \mathrm{H} \times 320 \mathrm{~V}$-element InSb (Indium - Antimonide) focal plane array detector. The radiometer's noise equivalent temperature difference $(\mathrm{NE} \Delta \mathrm{T})$, cited by the manufacturer, is $0.025^{\circ} \mathrm{C}$ when operating the detector in the 3 to 5 micrometer wavelength range. The radiometer produces images at both 30 frames per second output (video frame rate, in an RS170, format compatible with standard video equipment) and 60 frames per second output in a 14-bit, RS422 digital format. External optics, consisting of a wideangle lens, using germanium optical elements, were used to increase the system field-of-view by a factor of approximately two. The expanded field-of-view of this lens is $41^{\circ}$ horizontally and $31^{\circ}$ vertically. Heat application is achieved by directing the output of two 2400 Joule xenon flash lamps contained within a hood assembly that helps to focus the energy onto the inspection surface. 
Quantitative time based analysis requires synchronization between the IR imager and the heat source. This synchronization is achieved by computer control of the application of heat and the data acquisition. All experiments performed on the RCC material consisted of flash heating and then acquired thermal data was acquired during the cool-down of the inspection area for a total of 14 seconds. Images were recorded at a frame rate of 60 frames per second. The camera / hood assembly was mounted on a photographic copy stand in order to maintain a consistent standoff between the hood the surface of $2.54 \mathrm{~cm}$. For all cases presented in this paper, the maximum surface temperature change of the specimen above ambient was less than $10^{\circ} \mathrm{C}$.

\section{One-dimensional analytic model}

To simulate the front surface temperature response of the RCC material to a flash heat input, a one dimensional, multi-layer model was developed to solve the heat equation. A classic Laplace transformation approach was taken to solve the heat equation [[6],[7]]. The heat equation in a one dimensional slab of finite thickness is:

$$
\frac{\partial^{2} T}{\partial x^{2}}=-\frac{1}{\alpha} \frac{\partial T}{\partial t}
$$

where $T$ is temperature, $t$ is time, $x$ is the dimension normal to the surface of the slab with $x=0$ being the slab face of interest and $\alpha$ is the thermal diffusivity. The heat flux, $\Phi$, is assumed for this model to be a Dirac heat pulse of total energy $Q$ and is applied at $x=0$ with an insulating back surface $(x=l)$. In the time domain this leads to the following boundry conditions:

$$
\begin{aligned}
& \Phi=Q \delta(t) \text { at } x=0 \text { and } \\
& \Phi=0 \text { at } x=l .
\end{aligned}
$$

Applying a Laplace transform to the heat equation yields:

$$
\frac{d^{2} \theta}{d x^{2}}=\frac{P}{\alpha} \theta
$$

where $P$ is the Laplace parameter and $\theta$ is given by:

$$
\theta=\int_{0}^{\infty} e^{-P t} T d t
$$

The boundary conditions can also be transformed into the Laplace domain as:

$$
\phi=Q \text { at } x=0 \text { and }
$$




$$
\phi=0 \text { at } x=l
$$

It is typical to write the four quantities of interest $\theta_{i}, \phi_{i}, \theta_{o}$, and $\phi_{0}$, the front face Laplace temperature and flux and the Laplace temperature and flux at $x=1$ respectively, in matrix form as:

$$
\left[\begin{array}{l}
\theta_{0} \\
\phi_{o}
\end{array}\right]=\left[\begin{array}{ll}
A & B \\
C & D
\end{array}\right]\left[\begin{array}{l}
\theta_{i} \\
\phi_{i}
\end{array}\right]
$$

where,

$$
\left[\begin{array}{ll}
A & B \\
C & D
\end{array}\right]=\left[\begin{array}{cc}
\cosh (k l) & \frac{1}{k Q} \sinh (k l) \\
Q k \sinh (k l) & \cosh (k l)
\end{array}\right]
$$

and

$$
k=\sqrt{P / \alpha} .
$$

Finally, a material of $\mathrm{n}$ layers can be expressed in matrix form, in the Laplace domain, as follows:

$$
\left[\begin{array}{l}
\theta_{0} \\
\phi_{0}
\end{array}\right]=\left[\begin{array}{cc}
1 & -R_{n+1} \\
0 & 1
\end{array}\right]\left[\begin{array}{cc}
A_{n} & B_{n} \\
C_{n} & D_{n}
\end{array}\right]\left[\begin{array}{cc}
1 & -R_{n} \\
0 & 1
\end{array}\right] \ldots\left[\begin{array}{cc}
A_{1} & B_{1} \\
C_{1} & D_{1}
\end{array}\right]\left[\begin{array}{cc}
1 & -R_{1} \\
0 & 1
\end{array}\right]\left[\begin{array}{l}
\theta_{i} \\
\phi_{i}
\end{array}\right],
$$

where $R_{n}$ is a contact resistance that can be added between any layer to simulate a defect such as an air gap that would reduce the rate of heat flow. Once the matrix has been established, then numeric methods were used to perform the inverse Laplace transform back into the time domain.

The RCC material used on the Space Shuttle is actually a layered structure consisting of Silicon Carbide $(\mathrm{SiC})$ on the front and back surfaces and reinforced carbon-carbon in the middle. Additionally, most specimens have other coatings of various types applied to the outer (front) surface. Because of the complex nature of this material a four layer model was chosen to approximate the thermal response. A unitary input heat flux (Q) was chosen for the model since the actual heat deposited on the front surface is unknown. Fig. 2 shows the front surface temperature as predicted by the analytic model compared to experimental data for 15 seconds of cooling after the initial heat pulse for undamaged RCC material and for a flat-bottom hole. The output of the model and the experimental results were both normalized for comparison. Fig. 3 shows the difference between the model and the experimental data, in the undamaged RCC case, as a function of time, indicating that agreement of better than $99 \%$ is achieved after 0.13 seconds of cooling. The difference observed 
over the flat-bottom hole can be partially attributed to a failure of the 1-D model to correctly account for two dimensional heat flow effects due to the presence of the defects.

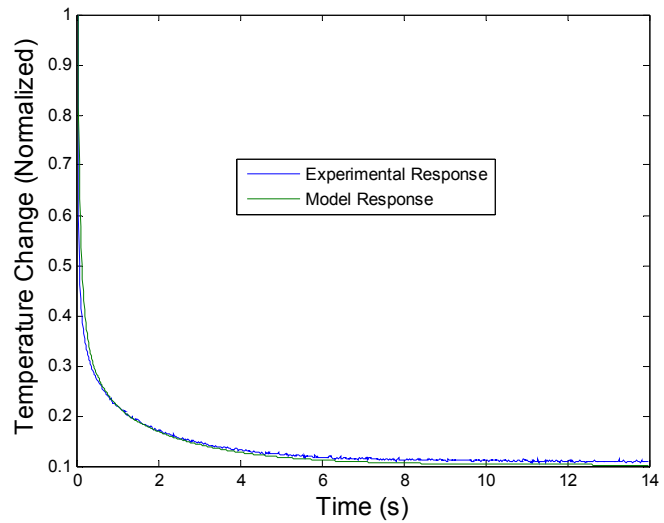

(a)

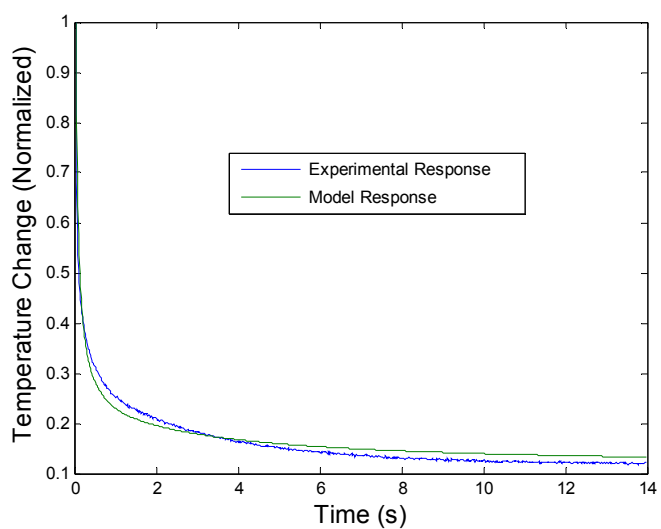

(b)

Fig. 2. Comparison of four layer 1-D analytic model results with the experimental response of RCC material for two situations (a) no defect and (b) a flatbottom hole. 


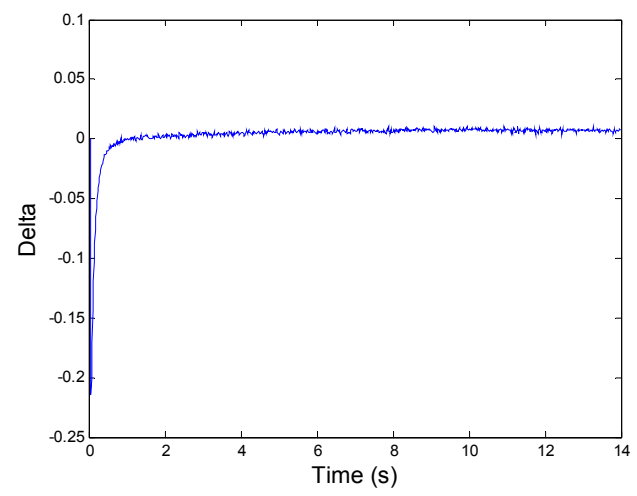

Fig. 3. Difference between experimental results and analytic model of undamaged RCC material showing better than $99 \%$ agreement after 0.13 seconds.

\section{Principal component analysis}

PCA is a common data reduction methodology applied to thermal NDE data. The algorithm is based on the decomposition of the thermal data into its principal components or eigen vectors using singular value decomposition (SVD). [3-5] PCA is performed by first reformatting the three-dimensional thermal data into a twodimensional array where the columns contain the spatial information and the rows contain the temporal information such that $T(x, y, t)$ becomes $A(n, m)$ where $n=N_{x}{ }^{*} N_{y}$ and $m=N_{t}$. The matrix $A$ is then adjusted by subtracting the mean along the time dimension, and decomposed to yield the eigen values and eigen vectors:

$$
A=U \Gamma V^{T}
$$

where $U$ and $V$ are orthogonal matrices who's columns form the eigenvectors of $A A^{\top}$ and $A^{\top} A$ respectively and $\Gamma$ contains the singular values (the nonnegative square roots of the eigen values) of $A^{\top} A$. Since the column of $U$ corresponding to nonzero singular values form an orthogonal basis for the range space of $A$, the entire thermal data set can be described by this basis. Because thermal NDE signals are well behaved and slowly varying in time, the predominant temporal variations of the entire data set are usually contained in the first or second eigenvector. The PCA images are formed by calculating the dot product of the measured temperature response, pixel by pixel, with the eigenvectors of interest (usually the two associated with the largest eigen values). Defects in RCC material change the local temporal variation of the data and thus appear as either light or dark regions in the PCA images.

While this process is quite effective in reducing thermal data, the SVD can be computationally intense especially with the very large three-dimensional arrays of thermal data typically produced in the inspection of RCC. In order to reduce the computation time involved, the results of the one-dimensional model of the RCC material with no defects was used to form the orthogonal basis. To provide a statistically rich data set for the calculation of the eigenvectors, a family of 10,000 
characteristic curve was produced using the one-dimensional model. The curves represent a series of thickness variations of $\pm 10 \%$ for each of the first three layers of the model. The resulting basis can then be used with any thermal inspection data to quickly form PCA images as described above.

\section{Experimental results}

The fixed eigen vector approach was applied to thermal inspection data from the Nose Cap of OV-105 (Space Shuttle Discovery) acquired at Lockheed Martin Missiles and Fire Control, Dallas, TX. Flash thermography was performed on this specimen using the EchoTherm ${ }^{\circledR}$ system. Data was collected at a frame rate of 60 frames per second for 14 seconds after the flash heating. Figure 4 shows the thermal inspection being performed. During processing refurbishment, visible damage was found on the nose cap dome around the T-seal lock-side surface; most notably two areas of significant $\mathrm{SiC}$ loss were visible. The damaged $\mathrm{SiC}$ areas measure approximately $1.9 \mathrm{~cm}$ high $\times 5.1 \mathrm{~cm}$ wide each; with visible $\mathrm{SiC}$ coating cracking surrounding the primary damage as seen in Figure 5 . It was confirmed using infrared thermography and PCA analysis that delaminations initiated at these sites and spread laterally along the circumference of the dome up to 18.4 centimeters. Figure 6 shows the second projection late time window (1 to 11.5 seconds after heating) fixed eigen vector PCA image of the regions around the visible SiC loss.

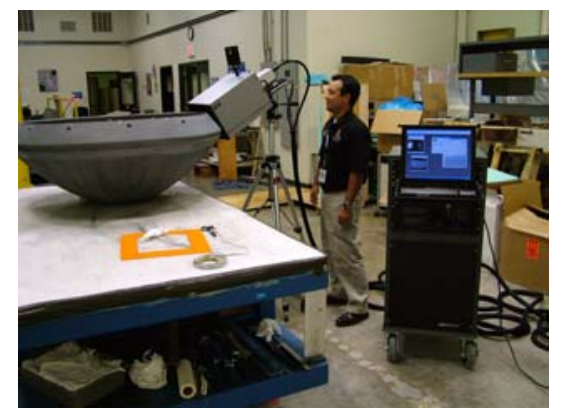

Fig. 4. Photograph of OV-105 Nose Cap inspection being performed by NASA LaRC personnel at Lockheed Martin Missile and Fire Control facilities in Dallas, $T X$.

\section{Conclusions}

A new approach to PCA processing of thermal NDE data has been presented that uses a fixed set of eigen vectors generated from a one dimensional analytic model of the thermal response of RCC material. Application of this approach to data acquired from a flat-bottom hole specimen showed that an increase in the signal-tonoise ratio of the resulting images can be achieved over conventional PCA. Further, this technique was found to reduce the processing time required to analyze typical thermal data sets. For a set of thermal data containing 850 frames, full PCA calculating two eigen vectors on one time window required on average 46.6 seconds 


\section{http://dx.doi.org/10.21611/qirt.2006.002}

in Matlab $^{\circledR}$. For the same size data set, using the fixed eigen vectors previously calculated from the one dimensional model, the processing time was reduced by a factor of approximately 2.2 to 21.0 seconds. This decrease in processing time becomes significant when applied to a complete inspection of the wing leading edges of the Space Shuttle. It is currently estimated that 600 data sets will be required to cover both wings during a thermal inspection. The use of fixed eigen vectors will reduced the processing time for this data by approximately 4.25 hours.

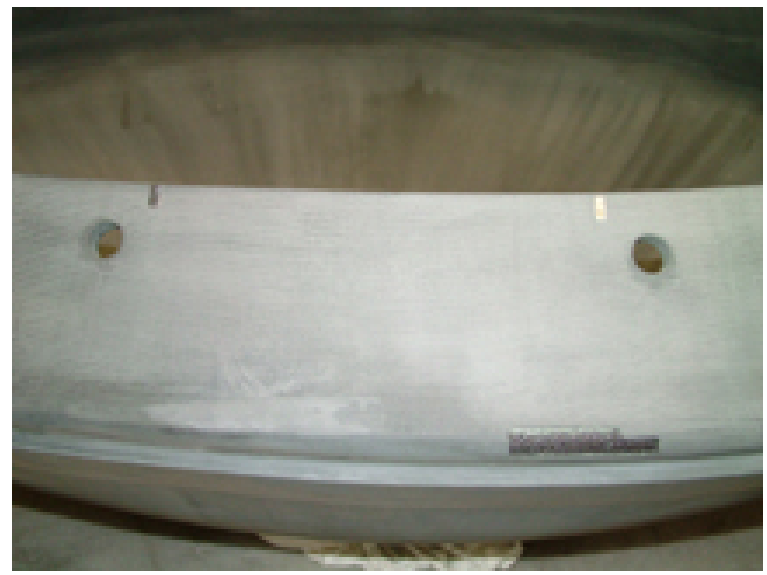

Fig. 5. Photographs of visible damage at two SiC loss regions on Nose Cap from OV-105.

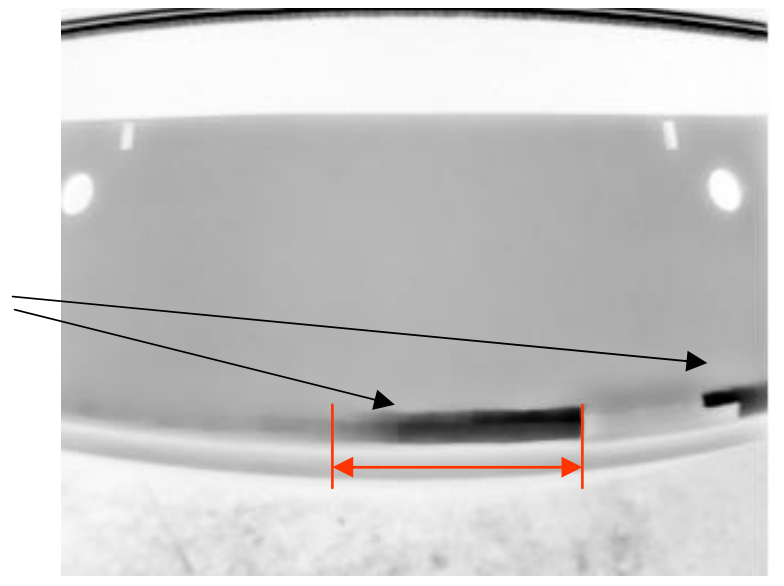

Fig. 6. Fixed eigen vector PCA image (second projection late time window) of the regions around the visible $\mathrm{SiC}$ loss showing an $11.4 \mathrm{~cm}$ long delamination. 


\section{REFERENCES}

[1] K.M. Joels and G.P. Kennedy, The Space Shuttle Operator's Manual, Ballantine Books, New York (1982).

[2] W.H. Gehman, et. al., "Columbia Accident Investigation Board," Report Vol 1, U.S. Government Printing Office, Washington, D.C. (2003).

[3] N. Rajic, "Principal Component Thermography for Flaw Contrast Enhancement and Flaw Depth Characterisation in Composite Structures," Composite Structures, 58 (2002) 521-528.

[4] N. Rajic "Principal Component Thermography," DSTO-TR-1298 (2002).

[5] J.N. Zalameda, P.A. Howell and W.P. Winfree, "Compression Techniques for Improved Algorithm Computational Performance," Proceedings of SPIE, Thermosense XXVII, 5782 (2005) 399-406.

[6] H.S. Carslaw and J.C. Jaeger, Conduction of Heat in Solids, Clarenon Press, Oxford (1986).

[7] D. Maillet, et. al., Thermal Quadrupoles, John Wiley \& Sons, Chichester (2000). 